\title{
A Microstep Controller of a DC Servomotor
}

\author{
KENZO WATANABE, SENIOR MEMBER, IEEE, AND HIROJI YOKOTE, STUDENT MEMBER, IEEE
}

\begin{abstract}
A microstep controller of a dc servomotor is developed for accurate positioning and smooth movement at low speed rotation. It consists of digital and analog positioning loops. Dividing one period of quadrature sinusoidal signals generated by an incremental encoder attached to a motor shaft into four quarter sections, the digital loop controls the movement between the sections. The analog loop divides each section further into $N$ equiangle segments, to control the movement within each section. The effective angle resolution is thus $90^{\circ} / M N$ with $M$ being the number of slits etched on an incremental encoder. A prototype controller assembled using monolithic and hybrid integrated components has confirmed the principles of operation. Experimental results are also presented to demonstrate its capabilities.
\end{abstract}

\section{INTRODUCTION}

A CLOSED-LOOP digital servo controller using a dc motor is now widely used, in place of an open-loop system using a stepping motor [1], for high speed accurate positioning in a quiet environment [2], [3]. A dominant factor that limits the positioning accuracy of such a dc servo controller is the angle resolution of an encoder attached to a motor shaft. The angle resolution of a conventional incremental encoder available at reasonable cost is $0.09^{\circ}$. Therefore, if a screw bolt with $4 \mathrm{~mm}$ pitch is used, a positioning accuracy of $1 \mu \mathrm{m}$ can be achieved. This accuracy is sufficient for most applications and the movement in high speed positioning is also smooth because of the motor inertia. In low speed operation, however, the intermittent movement due to the digital control becomes appreciable. This jogging movement is quite similar to that of a stepping motor, and thus could be improved by increasing the angle resolution of the encoder. Based on this idea, a microstep controller of a dc servomoter is developed.

A straightforward solution to increase the angle resolution is to increase the number of slits etched on a rotary disk. With current advancements in fine etching technologies, more than $10^{4}$ slits are possible. But, for an incremental encoder producible at reasonable cost, the number of slits is limited to 4000 and thus the angle resolution is limited to $0.0225^{\circ}$ if the multiply-by-four technique is used. Another solution is the use of an incremental encoder with quadrature sinusoidal outputs. Making use of the amplitude variation with angle, one can divide one period of the sinusoidal signal into $N$ equiangle segments to increase the effective angle resolution. This interpolation approach is adopted here because it relaxes the requirement for the encoder.

Manuscript received February 13, 1990; revised May 31, 1990.

The authors are with the Research Institute of Electronics, Shizuoka University, Hamamatsu 432, Japan.

IEEE Log Number 9038804.
Following these introductory remarks, the architecture, the principles of operation, and experimental results obtained by a prototype controller are described in subsequent sections.

\section{Architecture}

Fig. 1 shows the schematic diagram of the microstep controller. It consists of two main blocks; the digital loop for coarse positioning and the analog loop for fine positioning. The input command format is the same as that of a stepping motor. The target position and the velocity of the motor under control are specified, respectively, by the number and frequency of serial command pulses $\theta$. The motor shaft position is detected by an incremental encoder with quadrature sinusoidal outputs. Its output waveforms are depicted in Fig. 2. Receiving the sinusoidal signals $v_{a}$ and $v_{b}$ and their digital counterparts $\phi_{a}$ and $\phi_{b}$, the waveform converter in the analog loop divides one period of the sinusoidal signal into four quarter sections and produces the quasi-ramp signal $v_{r p}$, with a positive slope when the motor is rotating clockwise and a negative slope when rotating counterclockwise, in each quarter period.

The input command pulse $\theta$ is applied to the up/down counter in the analog loop, to increment or decrement it depending on the rotating direction command $\mathrm{CW} / \mathrm{CCW}$. The content of the up/down counter addresses the ROM which stores the digital codes to interpolate one quarter period of $v_{r p}$ into $N$ equiangle segments. Sent to the DAC, this code is converted to the analog reference voltage $v_{\text {ref }}$ with which $v_{r p}$ generated by the waveform converter is compared. The difference between $v_{\text {ref }}$ and $v_{r p}$ is fed through the driver and back to the motor to drive it to the specified position. It should be noticed here that the analog loop operates as a sampled-analog controller, in fact, and thus $v_{r p}$ is a staircase waveform in accord with $v_{\text {ref }}$, as shown in Fig. 3.

Assuming that the sampling frequency of the DAC is much higher than that of the input command $\theta$ and modeling the motor in terms of the inertia $J$ and the torque constant $K_{T}$, one can describe the transfer function $H_{a}(s)$ of the analog loop as follows:

$$
H_{a}(s)=\frac{\Phi(s)}{\Theta(s)}=\frac{K_{c}}{K_{s}} \cdot \frac{\omega_{a}^{2}}{s^{2}+2 \xi_{a} \omega_{a} s+\omega_{a}^{2}}
$$

where the natural frequency $\omega_{a}$ and the damping factor $\xi_{a}$ are given by

$$
\omega_{a}=\sqrt{\frac{K_{s} K_{a} K_{T}}{J}}
$$




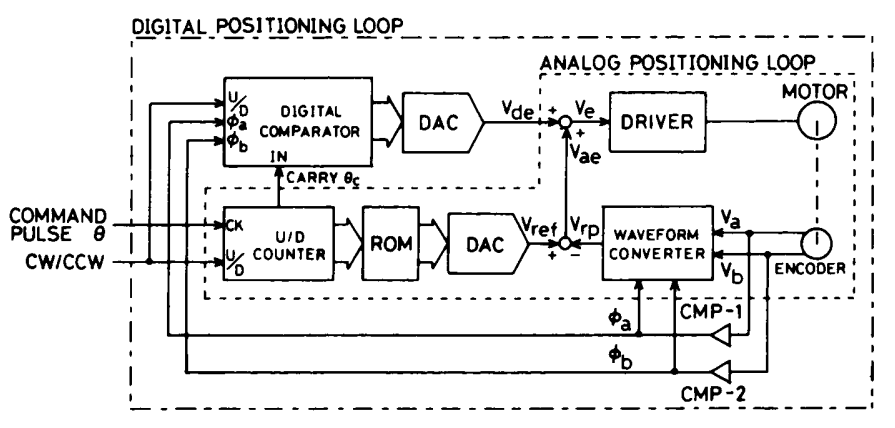

Fig. 1. Schematic diagram of the microstep controller.

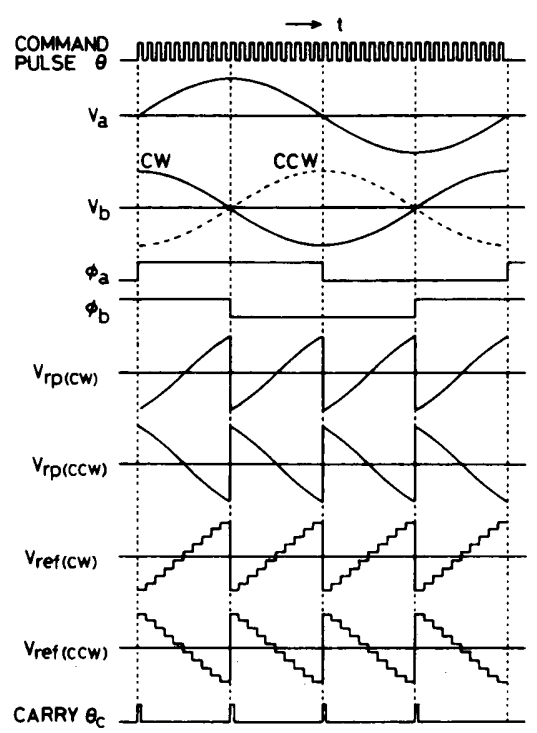

Fig. 2. Timing diagram of the analog and digital signals in the microstep controller.

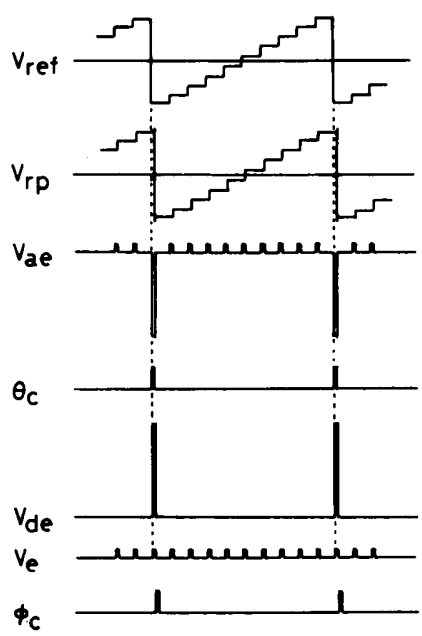

Fig. 3. Timing diagram of the error signals, the carry $\theta_{c}$, and the position index signal $\phi_{c}$.

and

$$
\xi_{a}=\frac{K_{d} \omega_{a}}{2}
$$

respectively, where $K_{c}$ is the conversion gain of the DAC in the analog loop, $K_{a}$ is the voltage-to-current transfer gain of the driver, and $K_{s}$ and $K_{d}$ are the proportional and derivative constants, respectively, of the waveform con- verter accommodating the proportional-derivative (PD) controller. Equation (1) indicates that if the loop gain $K_{s} K_{a} K_{T} / J$ is sufficiently high and $K_{c}=K_{s}$, then the actual motor position $\phi$ exactly tracks the input position command $\theta$ and thus rotates by $90^{\circ} / M N$ each time the analog loop receives $\theta$, where $M$ is the number of slits etched on the encoder. It should be noted here that the analog loop is valid only for the movement within one quarter section. The movement between the sections is controlled by the digital loop.

The up/down counter in the analog loop produces a carry each time it counts $N$, as shown in Fig. 2. Sent to the digital loop, this carry increments or decrements the up/down counter accommodated in the digital comparator, depending on the rotating direction command $\mathrm{CW} / \mathrm{CCW}$. The content of the up/down counter is then converted into the digital error signal $v_{d e}$ by the DAC and added to the analog error signal $v_{a e}$. The conversion gain of the DAC is chosen such that the digital error signal overcomes the maximum value of the analog error signal $v_{a e}$. This occurs when the motor traverses the quarter section as illustrated in Fig. 3. Thus the digital loop activated by a carry drives the motor to the next quarter section until the position index signal $\phi_{c}$, which is generated in the digital comparator by detecting rising and falling edges of $\phi_{a}$ and $\phi_{b}$, cancels the digital error signal $v_{d e}$. This transition is carried out instantly and the position control is left again to the analog loop.

Assuming again that the sampling frequency of the DAC is much higher than the carry frequency, one can approximate the transfer function $H_{d}(s)$ of the digital loop as follows:

$$
H_{d}(s)=\frac{\Phi(s)}{\Theta_{c}(s)}=\frac{K_{g}}{K_{g}+K_{s}} \cdot \frac{\omega_{d}^{2}}{s^{2}+2 \xi_{d} \omega_{d} s+\omega_{d}^{2}}
$$

where

$$
\begin{aligned}
& \omega_{d}=\sqrt{\frac{K_{a} K_{T}\left(K_{g}+K_{s}\right)}{J}} \\
& \xi_{d}=\frac{K_{d} K_{s}}{K_{g}+K_{s}} \cdot \frac{\omega_{d}}{2}
\end{aligned}
$$

and $K_{g}$ is the conversion gain of the DAC in the digital loop. It is clear from (4) that the waveform converter in the analog loop introduces damping into the digital loop and the position index signal $\phi_{c}$ follows closely the carry $\theta_{c}$ without a tracking error if the conversion gain $K_{g}$ of the DAC is much higher than that $K_{s}$ of the waveform converter.

Summarizing the previously stated analyses of the analog and digital loops, it is concluded that this controller performs stable, digital positioning of a dc motor with an accuracy of $90^{\circ} / M N$.

\section{Prototype Controller}

A prototype controller was assembled to confirm the principles of operation. For easy assembly, a CMOS chip 


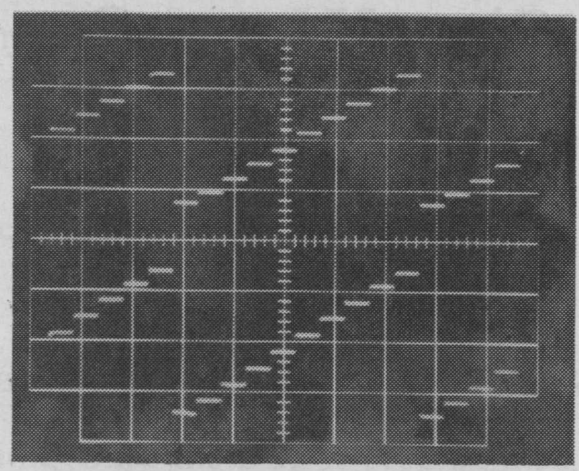

Fig. 4. Reference voltage $v_{\text {ref }}$ (upper trace) and motor response $v_{r p}$ (lower trace) when the $\mathrm{CW}$ position command of $1 \mathrm{pps}$ was applied to a prototype controller. Vertical scale: $2 \mathrm{~V} /$ div. Horizontal scale: $2 \mathrm{~s} /$ div.

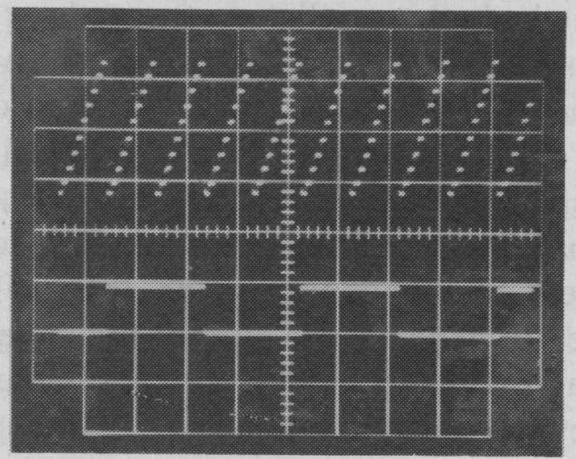

Fig. 5. Reference voltage $v_{\text {ref }}$ (upper trace) and $\phi_{a}$ (lower trace) observed in a prototype controller when the $\mathrm{CW}$ position command of $10 \mathrm{pps}$ was applied. Vertical scale: $2 \mathrm{~V} / \operatorname{div}$ (upper trace); $5 \mathrm{~V} /$ div (lower trace). Horizontal scale: $1 \mathrm{~s} /$ div.

developed for position and velocity control of the dc motor was used for the digital comparator. The chip includes synchronous differentiators to produce the position index signal $\phi_{c}$ and an 8-b up/down counter to compare $\theta_{c}$ with $\phi_{c}$. Its details are described elsewhere [4]. A key component in the analog loop is the waveform converter. This converter, based on a differential amplifier, was also integrated into a hybrid IC form using surface mount technologies. Its circuit configuration is described in [5]. The others are off-the-shelf components; a 4-b up/down counter MC14510B, an 8-b ROM, and a DAC-08 for the analog loop and another DAC-08 for the digital loop. The ROM stores the digital codes to interpolate the one quarter section into 10 equiangle segments. The driver consists of the pulsewidth modulator followed by the power bridge. Its switching frequency is $25 \mathrm{kHz}$.

Experiments were carried out using an unloaded $50-\mathrm{W}$ dc servomotor. An optical encoder with 1000 slits for each phase was used to detect the motor shaft position. Since $M=1000$ and $N=10$, the effective angle resolution is $0.009^{\circ}$ and the motor makes one revolution by 40000 steps.

Fig. 4 shows the motor response when the $\mathrm{CW}$ position command of 1 pps was applied to a prototype controller. It can be seen that $v_{r p}$ in the lower trace, the sampled- analog version of the quasi-ramp waveform in Fig. 2, agrees exactly with $v_{\text {ref }}$ in the upper trace. This confirms that the motor position tracks the position command without any delay. The motor speed was $1.5 \times 10^{-3} \mathrm{rpm}$. Even at such a low speed, the motor rotated smoothly in analog fashion.

Fig. 5 shows the motor response when the frequency of the position command $\theta$ was increased to $10 \mathrm{pps}$. The phase relation between $v_{r p}$ (upper trace) and $\phi_{a}$ (lower trace) indicates that one period of the sinusoidal output of the encoder is divided into four quarter sections and each section is further divided into 10 equiangle segments. These experimental results demonstrate the utility of the micro-step operation of a dc motor. The step angle is $0.009^{\circ}$, but it has an inaccuracy estimated to be $\pm 0.002^{\circ}$ due to the amplitude fluctuation of the encoder outputs.

\section{CONCLUSIONS}

A servo controller for microstep operation of a dc motor has been described. Experimental results obtained by a prototype controller have also been presented to demonstrate that the microstep operation based on the interpolation is very useful for fine positioning and smooth rotation at low velocity. Positioning accuracy of a prototype controller was $0.009^{\circ} \pm 0.002^{\circ}$. A further improvement is possible with this controller by increasing the number of slits on the encoder and reducing the interpolation step size. Positioning accuracy is dominated ultimately by the amplitude fluctuation of quadrature sinusoidal outputs and thus an automatic amplitude control should be incorporated into the encoder.

Crystal-pulling apparatus, astronomical observation systems, and tracking radars require smoothly rotating mechanisms. The microstep controller presented herein was first intended for such applications, but it can also be applied to fine positioning mechanisms in scanning electron and tunneling microscopes and semiconductor manufacturing equipment.

\section{ACKNOWLEDGMENT}

The authors wish to thank M. Suzuki of JR Tokai for his help in this experimental work.

\section{REFERENCES}

[1] T. R. Fredriksen, "Applications of the closed loop stepping motor,' IEEE Trans. Automat. Cont., vol. AC-13, pp. 464-474, 1968.

[2] B. K. Bose, Ed., Microcomputer Control of Power Electronics and Drives. New York: IEEE Press, 1987

[3] D. Squires, T. Isbell, and J. Santos, "Closed loop motion micro-controller: Overview, "' in Proc. Applied Motion Control Conf., pp. 173$179,1986$.

[4] K. Watanabe, A. Suzuki, K. Mutoh, and Y. Yamazaki, "A CMOS chip for position and speed control of a dc motor," in Proc. Applied Motion Control Conf., p. 251, 1987.

[5] H. Yokote and K. Watanabe, "A hybrid digital and analog controller for dc and brushless servomotors," IEEE Trans. Instrum. Meas., vol. 39 , pp. $259-263$, Feb. 1990 